Pacific

Journal of

Mathematics

APPROXIMATION WITH NORMAL OPERATORS WITH FINITE SPECTRUM, AND AN ELEMENTARY PROOF OF A BROWN-DOUGLAS-FILLMORE THEOREM

Peter Friss and Mikael Rørdam 


\title{
APPROXIMATION WITH NORMAL OPERATORS WITH FINITE SPECTRUM, AND AN ELEMENTARY PROOF OF A BROWN-DOUGLAS-FILLMORE THEOREM
}

\author{
Peter Friss and Mikael Rørdam
}

\begin{abstract}
We give a short proof of the theorem of Brown, Douglas and Fillmore that an essentially normal operator on a Hilbert space is of the form "normal plus compact" if and only if it has trivial index function. The proof is basically a modification of our short proof of Lin's theorem on almost commuting selfadjoint matrices that takes into account the index.

Using similar methods we obtain new results, generalizing results of Lin, on approximating normal operators by ones with finite spectrum.
\end{abstract}

\section{Introduction.}

Let $H$ be an infinite-dimensional separable Hilbert space, let $\mathcal{K}$ denote the compact operators on $H$, and consider the short-exact sequence

$$
0 \longrightarrow \mathcal{K} \longrightarrow B(H) \stackrel{\pi}{\longrightarrow} Q(H) \longrightarrow 0
$$

where $Q(H)$ is the Calkin algebra $B(H) / \mathcal{K}$. An operator $T \in B(H)$ is essentially normal if $T^{*} T-T T^{*} \in \mathcal{K}$, or equivalently, if $\pi(T)$ is normal.

An operator $T \in B(H)$ is Fredholm if $\pi(T)$ is invertible in $Q(H)$, and it's Fredholm index is denoted by $\operatorname{index}(T)$. The essential spectrum $\operatorname{sp}_{\text {ess }}(T)$ is the spectrum of $\pi(T)$. The index function of $T$ is the map

$$
\mathbb{C} \backslash \operatorname{sp}_{\text {ess }}(T) \rightarrow \mathbb{Z} ; \quad \lambda \mapsto \operatorname{index}(T-\lambda \cdot 1) .
$$

The index function is invariant under compact perturbations. Hence we may define the index function of an invertible $S \in Q(H)$ to be that of any lift $T \in B(H)$ of $S$.

The index function is continuous and hence constant on each connected component of its domain. It vanishes on the unbounded connected component of its domain. We say that an operator has trivial index function if its index function is zero everywhere on its domain.

In Section 2 we give a new proof of the following:

Theorem 1.1 ([BDF1, Cor. 11.2]). An essentially normal operator on a Hilbert space is a compact perturbation of a normal operator if and only if it has trivial index function. 
The theorem is a special case of the result by Brown-Douglas-Fillmore ([BDF1, Theorem 11.1]) that two essentially normal operators are unitarily equivalent modulo a compact perturbation if and only if they have the same essential spectrum and index function. In fact the general theorem is a fairly straightforward consequence of Theorem 1.1, see [BD, Theorem 5.8] and [D, Proposition 4.1].

Our proof of Theorem 1.1 has two steps. The first step is to show that an essentially normal operator with trivial index function is in the closure of the set of compact perturbations of normal operators on $H$. We prove this by showing that a normal element in the Calkin algebra can be approximated by normal elements with finite spectra if it has trivial index function (see also Lin [L2]). The methods used here follow closely the methods we used in $[\mathbf{F R}]$. The main difference is that we here must keep track of the index (or $K_{1}$-class) of the invertible operators used in the various approximation steps. Step two is then to show that the set of compact perturbations of normal operators is norm closed. This step involves quasidiagonal essentially normal operators, and its proof is a straightforward consequence of Lin's Theorem:

Theorem 1.2 (H. Lin, [L2]). For every $\varepsilon>0$ there is a $\delta>0$ such that for every finite dimensional $C^{*}$-algebra $A$ and every element $T \in A$ such that

$$
\|T\| \leq 1 \quad \text { and } \quad\left\|T^{*} T-T T^{*}\right\|<\delta
$$

there is a normal element $N \in A$ such that $\|T-N\|<\varepsilon$.

See also the short proof of Lin's theorem in $[\mathbf{F R}]$.

In Section 3 we consider the general problem of approximating normal elements of a $\mathrm{C}^{*}$-algebra by normal elements with finite spectra.

We show that a normal element $a$ of a unital $\mathrm{C}^{*}$-algebra $A$ of real rank zero can be approximated by normal elements with finite spectra if and only if all its translates, $a-\lambda \cdot 1, \lambda \in \mathbb{C}$, belong to the closure of $\mathrm{GL}_{0}(A)$, the connected component of the identity in $\operatorname{GL}(A)$ (Theorem 3.2). A key step towards this end is that a normal element $a$ in any unital $\mathrm{C}^{*}$-algebra $A$ can be approximated by normal elements $b \in A$, with 1-dimensional spectra and with $b-\lambda \cdot 1 \in \mathrm{GL}_{0}(A)$ for all $\lambda$ not in the spectrum of $b$, if and only if all translates $a-\lambda \cdot 1$ belong to the closure of $\mathrm{GL}_{0}(A)$. To complete the argument we use a theorem of Lin ([L3, Theorem 5.4]) that every normal element with 1-dimensional spectrum in a $\mathrm{C}^{*}$-algebra of real rank zero is the norm-limit of normal elements with finite spectra.

In Corollary 3.12 to Theorem 3.2 it is shown that if $A$ is a unital $\mathrm{C}^{*}$ algebra of real rank zero and stable rank one, then every normal element $a \in A$, satisfying $\pi(a)-\lambda \cdot 1 \in \mathrm{GL}_{0}(A / I)$ for all proper ideals $I$ of $A$ and for all $\lambda \in \mathbb{C} \backslash \operatorname{sp}(\pi(a))$, can be approximated by normal elements with finite spectra. This result should be compared with the theorem of Lin in $[\mathbf{L} 4]$ 
which says that in a simple $\mathrm{C}^{*}$-algebra $A$ of real rank zero and with property (IR) - a property, considered in [FR], which is weaker than stable rank one - every normal element $a$, with $a-\lambda \cdot 1 \in \mathrm{GL}_{0}(A)$ for every $\lambda \in \mathbb{C} \backslash \operatorname{sp}(a)$, is the norm-limit of normal operators with finite spectra.

The first operator theoretic proof of Theorem 1.1 is due to Berg and Davidson $[\mathrm{BD}]$. In fact, their analysis gives a stronger quantitative version which, subject to a natural resolvent condition on the operator $T$, gives a bound on the norm of the compact perturbation in terms of the norm of the self-commutator $T^{*} T-T T^{*}$.

Also let us mention that Lin has generalized Theorem 1.1 to essentially normal elements of $M(A) / A$ for certain AF-algebras $A$ (see [L5]).

We thank Larry Brown for several suggestions that helped improve our results and our exposition.

\section{Proof of Theorem 1.1.}

The main part of the proof of Theorem 1.1 consists of showing the theorem below, which - as indicated - has already been proved by Huaxin Lin (noting that $Q(H)$ is purely infinite and simple). The proof presented here, we believe is shorter and more direct than Lin's proof.

Theorem 2.1 (cf. H. Lin, [L3, Theorem 4.4]). Let $T$ be a normal element in $Q(H)$. Then $T$ is the norm limit of a sequence of normal elements in $Q(H)$ with finite spectra if and only if $T$ has trivial index function.

The lemmas below serve to prove the "if"-part of the theorem.

Define the continuous function $f_{\varepsilon}: \mathbb{R}^{+} \rightarrow \mathbb{R}^{+}$by $f_{\varepsilon}(t)=\max \{t-\varepsilon, 0\}$. Let $B(\lambda, r)$ denote the closed disc in the complex plane with center $\lambda$ and radius $r$. We let $\mathbb{D}$ denote the unit disc $B(0,1)$, and $\mathbb{T}$ will be the unit circle.

Lemma 2.2. Let $T$ be a normal element in $Q(H)$, let $\lambda \in \operatorname{sp}(T)$ and let $\varepsilon>0$. There exists a normal element $S \in Q(H)$ with $\|T-S\| \leq 2 \varepsilon$, $\lambda \notin \operatorname{sp}(S)$, index $(S-\lambda \cdot 1)=0$, and

$$
\operatorname{sp}(S) \backslash B(\lambda, \varepsilon)=\operatorname{sp}(T) \backslash B(\lambda, \varepsilon) .
$$

Proof. We may without loss of generality assume that $\lambda=0$. Let $R \in$ $B(H)$ be any lift of $T$, and let $R=V|R|$ be the polar decomposition of $R$. The operator $V f_{\varepsilon}(|R|)$ and its adjoint, $V^{*} f_{\varepsilon}\left(\left|R^{*}\right|\right)$, have infinite-dimensional kernels (because $\pi(|R|)=|T|=\pi\left(\left|R^{*}\right|\right)$ is non-invertible). Let $W f_{\varepsilon}(|R|)$ be the polar decomposition of $V f_{\varepsilon}(|R|)$. The argument above shows that $1-W W^{*}$ and $1-W^{*} W$ are both infinite-dimensional projections. Hence $W$ extends to a unitary $U \in B(H)$ with $V f_{\varepsilon}(|R|)=U f_{\varepsilon}(|R|)$.

Notice that $\pi\left(V f_{\varepsilon}(|R|)\right)=\pi(V) f_{\varepsilon}(|T|)=\pi(U) f_{\varepsilon}(|T|)$ is normal (because $\pi(V)$ commutes with $|T|)$. This implies that $\pi(U)$ commutes with $f_{\varepsilon}(|T|)$, 
and therefore $S=\pi(U)\left(f_{\varepsilon}(|T|)+\varepsilon \cdot 1\right)$ is normal. Clearly, $S$ is invertible, and $S$ lifts to the invertible operator $U\left(f_{\varepsilon}(|R|)+\varepsilon \cdot 1\right)$, and so index $(S)=0$. The distance between $S$ and $T$ is estimated by

$$
\begin{aligned}
\|T-S\| \leq \| & \left\|(V)|T|-\pi(V) f_{\varepsilon}(|T|)\right\| \\
& \quad+\left\|\pi(U) f_{\varepsilon}(|T|)-\pi(U)\left(f_{\varepsilon}(|T|)+\varepsilon \cdot 1\right)\right\| \\
& \leq 2 \varepsilon .
\end{aligned}
$$

Let $E \in Q(H)^{* *}$ be the spectral projection for $|T|$ corresponding to the interval $[0, \varepsilon]$. Since $|S|=f_{\varepsilon}(|T|)+\varepsilon \cdot 1$, it follows that $E$ is the spectral projection of $|S|$ corresponding to $\{\varepsilon\}$ in $Q(H)^{* *}$. Since $T$ and $S$ are normal, $E$ commutes with $T$ and $S$ (being a Borel function of $T$ and of $S$ ). We therefore have

$$
\begin{aligned}
& \operatorname{sp}(T) \cup\{0\}=\operatorname{sp}(T E) \cup \operatorname{sp}(T(1-E)), \text { and } \\
& \operatorname{sp}(S) \cup\{0\}=\operatorname{sp}(S E) \cup \operatorname{sp}(S(1-E)) .
\end{aligned}
$$

To complete the argument, notice that $|T|(1-E)=\left(f_{\varepsilon}(|T|)+\varepsilon \cdot 1\right)(1-E)=$ $|S|(1-E)$, and hence $T(1-E)=S(1-E)$. Also, $\|T E\|=\||T| E\| \leq \varepsilon$ and $\|S E\|=\||S| E\| \leq \varepsilon$, which entails that $\operatorname{sp}(T F)$ and $\operatorname{sp}(S F)$ are both contained in $B(0, \varepsilon)$.

Lemma 2.3. Let $F$ be a finite subset of $\mathbb{C}$. The set of elements $S \in Q(H)$, satisfying $\operatorname{sp}(S) \cap F=\emptyset$ and $\operatorname{index}(S-\lambda \cdot 1)=0$ for all $\lambda \in F$, is open.

Proof. The set in question is a finite intersection of open sets of the form $\mathrm{GL}_{0}(Q(H))+\lambda \cdot 1$.

Lemma 2.4. Let $T \in Q(H)$ be normal with trivial index function, let $F$ be a finite subset of $\mathbb{C}$, and let $\varepsilon>0$. Then there exists a normal element $S \in Q(H)$ such that $\|T-S\| \leq \varepsilon, \operatorname{sp}(S) \cap F=\emptyset$, and

$$
\operatorname{index}(S-\lambda \cdot 1)=0 \text {, }
$$

for all $\lambda \in F$.

Proof. For some $0 \leq k \leq n$ we can write $F=\left\{\lambda_{1}, \lambda_{2}, \ldots, \lambda_{n}\right\}$ so that $F \cap \operatorname{sp}(T)=\left\{\lambda_{k+1}, \lambda_{k+2}, \ldots, \lambda_{n}\right\}$. By assumption, index $\left(T-\lambda_{j} \cdot 1\right)=0$ for each $j \leq k$.

Using Lemmas 2.2 and 2.3 we find successively normal elements $T_{k}=T$, $T_{k+1}, T_{k+2}, \ldots, T_{n}$ in $Q(H)$ satisfying

- $\left\|T_{j}-T_{j-1}\right\| \leq \varepsilon /(n-k)$,

- $\lambda_{1}, \lambda_{2}, \ldots, \lambda_{j} \notin \operatorname{sp}\left(T_{j}\right)$,

- index $\left(T_{j}-\lambda_{i} \cdot 1\right)=0$, for $i=1,2, \ldots, j$, and

- $\lambda_{j+1}, \lambda_{j+2}, \ldots, \lambda_{n} \in \operatorname{sp}\left(T_{j}\right)$.

Finally, $S=T_{n}$ will be as desired. 
For each $\varepsilon>0$ consider the $\varepsilon$-grid $\Gamma_{\varepsilon}$ in $\mathbb{C}$ defined by

$$
\Gamma_{\varepsilon}=\{x+i y \in \mathbb{C} \mid x \in \varepsilon \mathbb{Z} \quad \text { or } \quad y \in \varepsilon \mathbb{Z}\} .
$$

Lemma 2.5. Let $T \in Q(H)$ be normal with trivial index function, and let $\varepsilon>0$. Then there exits a normal element $S \in Q(H)$ with trivial index function, $\operatorname{sp}(S) \subseteq \Gamma_{\varepsilon}$, and such that $\|S-T\| \leq \varepsilon$.

Proof. Choose $N \in \mathbb{N}$ such that $N \varepsilon \geq\|T\|+\varepsilon / 4$. Put

$\Sigma_{\varepsilon}=\left\{x+i y \in \mathbb{C} \mid x, y \in \varepsilon\left(\mathbb{Z}+\frac{1}{2}\right)\right\}, \quad X=\{a+i b:|a| \leq N \varepsilon,|b| \leq N \varepsilon\}$.

Applying Lemma 2.4 to the finite set $\Sigma_{\varepsilon} \cap X$ we get a normal operator $S^{\prime} \in Q(H)$ satisfying: $\left\|S^{\prime}-T\right\| \leq \varepsilon / 4, \operatorname{sp}\left(S^{\prime}\right) \cap\left(\Sigma_{\varepsilon} \cap X\right)=\emptyset$, and $\operatorname{index}\left(S^{\prime}-\right.$ $\lambda \cdot 1)=0$ for all $\lambda \in \Sigma_{\varepsilon} \cap X$. Note that $\operatorname{sp}\left(S^{\prime}\right) \subseteq X \backslash \Sigma_{\varepsilon}(=Y)$.

There is a continuous path $t \mapsto f_{t}, t \in[0,1]$, of continuous functions $f_{t}: Y \rightarrow Y$ so that

- $f_{0}(z)=z$ for all $z \in Y$,

- $f_{1}(Y) \subseteq \Gamma_{\varepsilon}$,

- $f_{t}(z)=z$ for all $z \in \Gamma_{\varepsilon} \cap X$ and for all $t$,

- $\left|f_{1}(z)-z\right|<(\sqrt{2} / 2) \varepsilon$ for all $z \in Y$.

Put $S=f_{1}\left(S^{\prime}\right)$. Then $S$ is normal, $\operatorname{sp}(S) \subseteq \Gamma_{\varepsilon} \cap X$, and $\|T-S\| \leq$ $\varepsilon / 4+(\sqrt{2} / 2) \varepsilon \leq \varepsilon$.

If $\lambda \in \mathbb{C} \backslash \operatorname{sp}(S)$, then $\lambda$ is in the same connected component of $\mathbb{C} \backslash \operatorname{sp}(S)$ as some $\lambda^{\prime} \in \Sigma_{\varepsilon} \cap X$, or $\lambda$ is in the unbounded component of $\mathbb{C} \backslash \operatorname{sp}(S)$. In the latter case, $\operatorname{index}(S-\lambda \cdot 1)=0$, and in the former case,

$$
\begin{aligned}
\operatorname{index}(S-\lambda \cdot 1) & =\operatorname{index}\left(S-\lambda^{\prime} \cdot 1\right)=\operatorname{index}\left(f_{1}\left(S^{\prime}\right)-\lambda^{\prime} \cdot 1\right) \\
& =\operatorname{index}\left(f_{0}\left(S^{\prime}\right)-\lambda^{\prime} \cdot 1\right)=\operatorname{index}\left(S^{\prime}-\lambda^{\prime} \cdot 1\right)=0 .
\end{aligned}
$$

The lemma below is a special case of the Alexander Duality theorem from topology. For a compact subset $X$ of $\mathbb{C}$ it says that $\pi^{1}(X) \cong H^{1}(X ; \mathbb{Z}) \cong$ $H_{0}(\mathbb{C} \backslash X ; \mathbb{Z})$, and the latter is the free Abelian group generated by the bounded connected components of $\mathbb{C} \backslash X$.

Lemma 2.6. Let $X$ be a compact subset of $\Gamma_{\varepsilon}$ for some $\varepsilon>0$. Every continuous map $f: X \rightarrow \mathbb{C} \backslash\{0\}$ is homotopic to a map of the form

$$
z \mapsto\left(z-\lambda_{1}\right)^{n_{1}}\left(z-\lambda_{2}\right)^{n_{2}} \cdots\left(z-\lambda_{k}\right)^{n_{k}}
$$

for some $\lambda_{i} \in \mathbb{C} \backslash X$ and some $n_{i} \in \mathbb{Z}$.

Proof. Choose $n \in \mathbb{N}$ such that $X \subseteq[-n \varepsilon, n \varepsilon]^{2}$, and put $Y=\Gamma_{\varepsilon} \cap[-n \varepsilon, n \varepsilon]^{2}$. Then $f$ extends to a continuous function $g: Y \rightarrow \mathbb{C} \backslash\{0\}$. (Indeed, by considering only one line-segment of $Y$ at a time, this follows from the elementary fact that if $X_{0}$ is a closed subset of the interval $[0,1]$ and if 
$f_{0}: X_{0} \rightarrow \mathbb{C} \backslash\{0\}$ is continuous, then $f_{0}$ extends to a continuous function $g_{0}:[0,1] \rightarrow \mathbb{C} \backslash\{0\}$.)

Let $C_{1}, C_{2}, \ldots, C_{k}$ be the bounded connected components of $\mathbb{C} \backslash Y$. Each $C_{i}$ is an open square, $k=4 n^{2}$, and

$$
[-n \varepsilon, n \varepsilon]^{2}=Y \cup C_{1} \cup C_{2} \cup \cdots \cup C_{k} .
$$

Let $\gamma^{i}$ be the closed curve $\overline{C_{i}} \backslash C_{i}$ oriented in the positive direction, and denote by $n_{i}$ the winding number of $g$ around $\gamma^{i}$.

Choose a $y_{i} \in C_{i}$ for each $i$. Put

$$
h(z)=\left(z-\lambda_{1}\right)^{-n_{1}}\left(z-\lambda_{2}\right)^{-n_{2}} \cdots\left(z-\lambda_{k}\right)^{-n_{k}} g(z), \quad z \in Y .
$$

The winding number of $h$ around $\gamma^{i}$ is zero for each $i$. Therefore $\left.h\right|_{\gamma^{i}}$ extends to a continuous function $\overline{C_{i}} \rightarrow \mathbb{C} \backslash\{0\}$ for each $i$. This shows that $h$ extends to a continuous function $\tilde{h}:[-n \varepsilon, n \varepsilon]^{2} \rightarrow \mathbb{C} \backslash\{0\}$. Since $[-n \varepsilon, n \varepsilon]^{2}$ is contractible, $\tilde{h}$ is homotopic to the constant function 1 inside $C\left([-n \varepsilon, n \varepsilon]^{2}, \mathbb{C} \backslash\{0\}\right)$. Hence $\left.\tilde{h}\right|_{X}=\left.h\right|_{X}$ is homotopic to 1 inside $C(X, \mathbb{C} \backslash\{0\})$. The claim of the lemma follows immediately from this.

Lemma 2.7 is a natural generalization of [FR, Lemma 2.3] and the proof requires only a simple modification using Lemma 2.6. For the reader's convenience we include the entire proof.

Lemma 2.7. Let $T$ be a normal element in $Q(H)$ with trivial index function and with $\operatorname{sp}(T) \subseteq \Gamma_{\varepsilon}$ for some $\varepsilon>0$. Suppose that $I$ is a relatively open subset of $\operatorname{sp}(T)$ which is homeomorphic to the open interval $(0,1)$. Then for each $\lambda_{0} \in I$ and for each $\delta>0$ there is a normal element $S$ in $Q(H)$ with trivial index function such that $\|S-T\| \leq \delta$, and $\operatorname{sp}(S) \subseteq \operatorname{sp}(T) \backslash\left\{\lambda_{0}\right\}$.

Proof. Choose one point in each bounded connected component of $\mathbb{C} \backslash \operatorname{sp}(T)$, and let $F$ be the finite set of all these points. If $S \in Q(H)$ satisfies $\operatorname{sp}(S) \subseteq$ $\operatorname{sp}(T)$, then $S$ has trivial index function if index $(S-\lambda \cdot 1)=0$ for all $\lambda \in F$. It follows from Lemma 2.3 that $S$ has these properties if $\|T-S\| \leq \delta_{0}$ for a sufficiently small $\delta_{0}>0$. We may assume that $\delta \leq \delta_{0}$, and the part of the statement regarding $S$ having trivial index function is then automatically taken care of.

Let $J$ be a relatively open subset of $I$ satisfying

$$
\lambda_{0} \in J \subseteq \bar{J} \subseteq I, \quad \operatorname{diam}(J) \leq \varepsilon .
$$

Let $f_{0}: I \rightarrow \mathbb{T} \backslash\{-1\}$ be a homeomorphism, and extend $f_{0}$ to $f: \operatorname{sp}(T) \rightarrow \mathbb{T}$ by setting $f(z)=1$ for all $z \in \operatorname{sp}(T) \backslash I$. Let $V$ be the unitary element $f(T)$ of $Q(H)$.

It follows from Lemma 2.6 that $f$ is homotopic, inside $C(\operatorname{sp}(T), \mathbb{C} \backslash\{0\})$, to the function $g: \operatorname{sp}(T) \rightarrow \mathbb{C} \backslash\{0\}$ given by

$$
g(z)=\left(z-\mu_{1}\right)^{n_{1}}\left(z-\mu_{2}\right)^{n_{2}} \cdots\left(z-\mu_{k}\right)^{n_{k}},
$$


for appropriate $\mu_{i} \in \mathbb{C} \backslash \operatorname{sp}(T)$ and $n_{i} \in \mathbb{Z}$. Hence $f(T) \sim_{h} g(T)$ inside $\mathrm{GL}(Q(H))$ and

$$
\operatorname{index}(f(T))=\operatorname{index}(g(T))=\sum_{i=1}^{k} n_{i} \cdot \operatorname{index}\left(T-\mu_{i} \cdot 1\right)=0 .
$$

This shows that $V=f(T)$ lifts to a unitary $U$ in $B(H)$. We may now proceed exactly as in [FR, Lemma 2.3] (see also [BDF1, Lemma 6.1]).

Let $E \in B(H)$ be the spectral projection for $U$ corresponding to the (relatively open) subset $f(J)$ of $\operatorname{sp}(U)$, and put $F=\pi(E)$. Then $F$ is a projection in $Q(H)$. We show below that $F$ commutes with $T$, and that

(a) $\quad \operatorname{sp}_{F Q(H) F}(T F) \subseteq \bar{J}, \quad \operatorname{sp}_{(1-F) Q(H)(1-F)}(T(1-F)) \subseteq \operatorname{sp}(T) \backslash J$.

Once this has been established, we can choose any $\lambda_{1} \in J \backslash\left\{\lambda_{0}\right\}$ and set

$$
S=\lambda_{1} F+(1-F) T .
$$

Then $S$ is normal (because $T$ and $F$ commute),

$$
\operatorname{sp}(T) \subseteq\left\{\lambda_{1}\right\} \cup \operatorname{sp}(T) \backslash J \subseteq \operatorname{sp}(T) \backslash\left\{\lambda_{0}\right\},
$$

and $\|S-T\|=\left\|T F-\lambda_{1} F\right\| \leq \operatorname{diam}(J) \leq \varepsilon$ as desired.

Suppose $\varphi: \operatorname{sp}(T) \rightarrow \mathbb{C}$ is a continuous function which is zero on $\operatorname{sp}(T) \backslash I$. Then

$$
\hat{\varphi}(z)=\left\{\begin{array}{cl}
\left(\varphi \circ\left(\left.f\right|_{I}\right)^{-1}\right)(z), & \text { if } z \in \mathbb{T} \backslash\{-1\} \\
0, & \text { if } z=-1
\end{array},\right.
$$

defines a continuous function $\mathbb{T} \rightarrow \mathbb{C}$ satisfying $\varphi=\hat{\varphi} \circ f$, and hence $\varphi(T)=$ $\hat{\varphi}(V)$. Since $E$ commutes with $U$, it follows that $F$ commutes with $V$ and hence with $\hat{\varphi}(V)=\varphi(T)$.

If $\varphi$ in addition is constant equal to 1 on $J$, then $\hat{\varphi}$ is constant equal to 1 on $f(J)$, which implies that $\varphi(T) F=F \varphi(T)=F$. If $\varphi$ instead vanishes on $\operatorname{sp}(T) \backslash J$, then $\hat{\varphi}$ vanishes on $\mathbb{T} \backslash f(J)$, whence $\varphi(T) F=F \varphi(T)=\varphi(T)$.

Let $h: \operatorname{sp}(T) \rightarrow[0,1]$ be a continuous function with $\left.h\right|_{J}=1$ and $\left.h\right|_{\operatorname{sp}(T) \backslash I}=$ 0 . By the argument above, $h(T) F=F h(T)=F$, and since the function $z \mapsto z h(z)$ vanishes on $\operatorname{sp}(T) \backslash I$, we get

$$
T F=T h(T) F=F T h(T)=F h(T) T=F T .
$$

To show (\$) it suffices to show that $\varphi(T F)=0$ and $\psi(T(1-F))=0$ for every pair of continuous functions $\varphi, \psi: \operatorname{sp}(T) \rightarrow \mathbb{C}$, where $\varphi$ vanishes on $\bar{J}$ and $\psi$ vanishes on $\operatorname{sp}(T) \backslash I$ (and where the continuous functions operate in the respective corner algebras). We may assume that $\varphi$ is equal to 1 on the set $\operatorname{sp}(T) \backslash I$. From the argument in the previous paragraph we get $\varphi(T F)=\varphi(T) F=F-(1-\varphi(T)) F=0, \psi(T(1-F))=\psi(T)(1-F)=0$, as desired. 
Proof of Theorem 2.1. Assume that $T_{n}$ is a sequence of normal elements of $Q(H)$ so that $T_{n}$ tends to $T$ in norm, and $T_{n}$ has finite spectrum for each $n$. Let $\lambda \in \mathbb{C} \backslash \operatorname{sp}(T)$. Then $\lambda \in \mathbb{C} \backslash \operatorname{sp}\left(T_{n}\right)$ for all sufficiently large $n$, and index $\left(T_{n}-\lambda \cdot 1\right) \rightarrow$ index $(T-\lambda \cdot 1)$. But index $\left(T_{n}-\lambda \cdot 1\right)=0$ because $\mathbb{C} \backslash \operatorname{sp}\left(T_{n}\right)$ is connected, so index $(T-\lambda \cdot 1)=0$. This shows that $T$ has trivial index function.

Assume now that $T$ is a normal element of $Q(H)$ with trivial index function, and let $\varepsilon>0$. By Lemma 2.5 there is a normal element $T^{\prime} \in Q(H)$ with $\operatorname{sp}\left(T^{\prime}\right) \subseteq \Gamma_{\varepsilon},\left\|T-T^{\prime}\right\| \leq \varepsilon$, and such that $T^{\prime}$ has trivial index function.

We show next that there exists a normal element $T^{\prime \prime} \in Q(H)$ such that

$$
\left\|T^{\prime}-T^{\prime \prime}\right\| \leq \varepsilon, \quad \operatorname{sp}\left(T^{\prime \prime}\right) \subseteq \Gamma_{\varepsilon},
$$

and such that $\operatorname{sp}\left(T^{\prime \prime}\right)$ contains no entire line segment of $\Gamma_{\varepsilon}$, where an entire line segment is a set of the form

$$
\{n+i y \mid m \varepsilon<y<(m+1) \varepsilon\} \quad \text { or } \quad\{x+i m \mid n \varepsilon<x<(n+1) \varepsilon\},
$$

for some $n, m \in \mathbb{Z}$.

Let $\left\{I_{1}, I_{2}, \ldots, I_{n}\right\}$ be the set of entire line segments of $\Gamma_{\varepsilon}$ that are contained in $\operatorname{sp}\left(T^{\prime}\right)$, and choose $\lambda_{j} \in I_{j}$ for each $j$. Apply Lemma 2.7 successively to obtain normal elements $R_{0}=T^{\prime}, R_{1}, R_{2}, \ldots, R_{n}$ in $Q(H)$ such that

$$
\left\|R_{j+1}-R_{j}\right\| \leq \varepsilon / n, \quad \operatorname{sp}\left(R_{j+1}\right) \subseteq \operatorname{sp}\left(R_{j}\right) \backslash\left\{\lambda_{j+1}\right\},
$$

and each with trivial index function. The element $T^{\prime \prime}=R_{n}$ then has the desired property.

It now follows that $\operatorname{sp}\left(T^{\prime \prime}\right)$ can be partitioned into finitely many clopen sets $C_{1}, C_{2}, \ldots, C_{m}$ each with diameter less than $2 \varepsilon$. Choose $\mu_{i} \in C_{i}$ for each $i$, and let $f: \operatorname{sp}\left(T^{\prime \prime}\right) \rightarrow\left\{\mu_{1}, \mu_{2}, \ldots, \mu_{m}\right\}$ be the continuous function which maps $C_{i}$ to $\mu_{i}$. Then $|f(z)-z|<2 \varepsilon$ for all $z \in \operatorname{sp}\left(T^{\prime \prime}\right)$. The element $S=f\left(T^{\prime \prime}\right)$ is normal with $\operatorname{sp}(S)=\left\{\mu_{1}, \mu_{2}, \ldots, \mu_{m}\right\}$, and $\left\|S-T^{\prime \prime}\right\| \leq 2 \varepsilon$, so that $\|S-T\| \leq 4 \varepsilon$.

Recall that an operator $T$ is quasidiagonal if there is an increasing sequence $\left\{E_{n}\right\}_{n=1}^{\infty}$ of finite rank projections converging strongly to 1 such that $\lim _{n \rightarrow \infty}\left\|T E_{n}-E_{n} T\right\|=0$. The set of quasidiagonal operators is invariant under compact perturbations and is norm closed. That the set is norm closed can be seen by using the equivalent "local" definition of $T$ being quasidiagonal, that for every finite rank projection $E \in B(H)$ and for every $\varepsilon>0$ there exists a finite rank projection $F \in B(H)$ such that $E \leq F$ and $\|T F-F T\| \leq \varepsilon$.

Every normal element $N$ with finite spectrum is quasidiagonal (write $N=\sum_{j=1}^{k} \lambda_{j} P_{j}$ and put $E_{n}=\sum_{j=1}^{k} F_{n}^{(j)}$, where $\left\{F_{n}^{(j)}\right\}_{n=1}^{\infty}$ is an increasing sequence of finite rank projections converging strongly to $P_{j}$ ). Since every 
normal operator in $B(H)$ can be approximated by normal operators with finite spectrum, every normal operator is in fact quasidiagonal.

The statements in the proposition below were consequences of Theorem 1.1 in [BDF1]. Here it is used as a step in our proof.

Proposition 2.8 (cf. [BDF1, Cor. 11.4 and Cor. 11.12]). The set of compact perturbations of normal operators on a Hilbert space $H$ is equal to the set of quasidiagonal essentially normal operators. In particular, the set of compact perturbations of normal operators operators on $H$ is norm-closed.

Proof. Each compact perturbation of a normal operator is clearly essentially normal, and - by the remarks above - also quasidiagonal.

It is well-known that every quasidiagonal operator $T \in B(H)$ is blockdiagonal plus compact, i.e., there exist $S \in B(H)$ and an increasing sequence $\left\{E_{n}\right\}_{n=1}^{\infty}$ of finite rank projections converging strongly to 1 such that $T-S \in \mathcal{K}$ and $S E_{n}=E_{n} S$ for all $n$. (Indeed, choose $\left\{E_{n}\right\}_{n=1}^{\infty}$ such that $\sum_{n=1}^{\infty}\left\|E_{n} T-T E_{n}\right\|<\infty$. Put $E_{0}=0$, and put $S=\sum_{n=1}^{\infty}\left(E_{n}-\right.$ $\left.E_{n-1}\right) T\left(E_{n}-E_{n-1}\right)$. Then

$$
T-S=\sum_{n=1}^{\infty}\left(E_{n}-E_{n-1}\right) T\left(1-E_{n}\right)+\sum_{n=1}^{\infty}\left(E_{n}-E_{n-1}\right) T E_{n-1},
$$

and the right-hand side is compact since the two terms are norm-convergent sums of compact operators.)

Assume now that $T$ is essentially normal and quasidiagonal. Notice that $S$, being a compact perturbation of $T$ is also essentially normal. Put

$$
S_{n}=\left(E_{n}-E_{n-1}\right) T\left(E_{n}-E_{n-1}\right) .
$$

Since $S=\sum_{n=1}^{\infty} S_{n}$, and $S S^{*}-S^{*} S$ is compact, we have

$$
\lim _{n \rightarrow \infty}\left\|S_{n} S_{n}^{*}-S_{n}^{*} S_{n}\right\|=0 .
$$

Each $S_{n}$ lies in the finite dimensional C*-algebra $B\left(H_{n}\right)$, where $H_{n}=\left(E_{n}-\right.$ $\left.E_{n-1}\right)(H)$, and so Lin's theorem (Theorem 1.2) says that there exist normal operators $R_{n} \in B\left(H_{n}\right)$ with $\lim _{n \rightarrow \infty}\left\|R_{n}-S_{n}\right\|=0$. Put $R=\sum_{n=1}^{\infty} R_{n}$. Then $R$ is normal, and $R-S$ is compact. This proves that $T$ is a compact perturbation of a normal operator.

The set of essentially normal operators and the set of quasidiagonal operators are both closed. Hence so is their intersection.

Proof of Theorem 1.1. A compact perturbation of a normal operator has trivial index function since this is the case for a normal operator and since the index function is invariant under compact perturbation.

Assume now that $T$ is essentially normal with trivial index function. Then $\pi(T) \in Q(H)$ is normal with trivial index function. Hence, by Theorem 2.1, there is a sequence $\left\{S_{n}\right\}_{n=1}^{\infty}$ of normal elements of $Q(H)$ with finite spectra such that $S_{n} \rightarrow \pi(T)$ in norm. Lift $S_{n}$ to $T_{n} \in B(H)$ such that $T_{n} \rightarrow T$ in 
norm. Every normal operator in $Q(H)$ with finite spectrum has a lift to a normal operator in $B(H)$. Hence we can find normal operators $R_{n} \in B(H)$ with $\pi\left(R_{n}\right)=S_{n}=\pi\left(T_{n}\right)$. It follows that each $T_{n}$ is a compact perturbation of a normal operator. By Proposition 2.8 this shows that $T$ itself is a compact perturbation of a normal operator.

Corollary 2.9. An essentially normal operator on a Hilbert space has trivial index function if and only if it is quasidiagonal.

Proof. Combine Theorem 1.1 with Proposition 2.8.

\section{Approximating normal elements with normal elements with finite spectra.}

In this section we prove various generalizations of Theorem 2.1 using more or less the same methods as in Section 2.

We first consider an obstruction - analogous to the index-obstruction in Theorem 2.1 - for a normal element of a $\mathrm{C}^{*}$-algebra to be a norm-limit of normal elements with finite spectra. The natural generalization of the index function of an element of $Q(H)$ to an element $a$ of a unital $\mathrm{C}^{*}$-algebra $A$ is the map

$$
\mathbb{C} \backslash \operatorname{sp}(a) \rightarrow K_{1}(A) ; \quad \lambda \mapsto[a-\lambda \cdot 1]_{1} .
$$

Proposition 3.1 below shows that we must also take into account the index function of $a$ in every quotient of $A$. For each proper ideal $I$ of $A$ (proper ideal meaning $I \neq A$ ) we must consider the maps

$$
\mathbb{C} \backslash \operatorname{sp}\left(\pi_{I}(a)\right) \rightarrow K_{1}(A / I) ; \quad \lambda \mapsto\left[\pi_{I}(a)-\lambda \cdot 1\right]_{1},
$$

where $\pi_{I}$ denotes the quotient mapping $A \rightarrow A / I$. This additional obstruction was hidden in the case of the simple $\mathrm{C}^{*}$-algebra $Q(H)$.

Proposition 3.1. Let $A$ be a unital $C^{*}$-algebra and let $a \in A$ be a normal element. If a is the norm limit of normal elements in $A$ with finite spectra, then

$$
\pi_{I}(a)-\lambda \cdot 1 \in \mathrm{GL}_{0}(A / I)
$$

for every proper ideal $I$ of $A$ and every $\lambda \in \mathbb{C} \backslash \operatorname{sp}\left(\pi_{I}(a)\right)$.

Lin showed in [L3] that the natural map $\mathrm{GL}(A) / \mathrm{GL}_{0}(A) \rightarrow K_{1}(A)$ is injective if $\operatorname{RR}(A)=0$. Since the property real rank zero passes to quotients, this shows that $(\diamond)$ could be replaced by

$$
\left[\pi_{I}(a)-\lambda \cdot 1\right]_{1}=0 \text { in } K_{1}(A / I)
$$

if $\operatorname{RR}(A)=0$. 
Proof. Suppose that $\pi_{I}(a)-\lambda \cdot 1 \notin \mathrm{GL}_{0}(A / I)$ for some proper ideal $I$ of $A$ and some $\lambda \in \mathbb{C} \backslash \operatorname{sp}\left(\pi_{I}(a)\right)$. Then $a$ belongs to the open set

$$
\left\{b \in A \mid \pi_{I}(b)-\lambda \cdot 1 \in \mathrm{GL}(A / I) \backslash \mathrm{GL}_{0}(A / I)\right\} .
$$

This set can contain no element with finite spectrum, because if $b$ was such an element, $\pi_{I}(b) \in A / I$ would have finite spectrum and then either $\pi_{I}(b)-\lambda \cdot 1$ is not invertible or belongs to $\mathrm{GL}_{0}(A / I)$ since $\mathbb{C} \backslash \operatorname{sp}\left(\pi_{I}(b)\right)$ is connected.

Next, we investigate to what extent the converse of Proposition 3.1 holds. Recall the definition of the $\varepsilon$-grid $\Gamma_{\varepsilon}$ given above Lemma 2.5.

Theorem 3.2. Let $A$ be a unital $C^{*}$-algebra and let a be a normal element in $A$. The following conditions are equivalent:

(i) $a-\lambda \cdot 1$ lies in the closure of $\mathrm{GL}_{0}(A)$ for every $\lambda \in \mathbb{C}$,

(ii) for every $\varepsilon>0$ there exists a normal element $b \in A$ such that

$$
\operatorname{sp}(b) \subseteq \Gamma_{\varepsilon}, \quad\|a-b\| \leq 2 \varepsilon, \quad b-\lambda \cdot 1 \in \mathrm{GL}_{0}(A)
$$

for all $\lambda \in \mathbb{C} \backslash \operatorname{sp}(b)$.

If the real rank of $A$ is zero, then (i) and (ii) are equivalent to

(iii) for every $\varepsilon>0$ there exists a normal element $b \in A$ with finite spectrum and with $\|a-b\| \leq \varepsilon$.

The implication (ii) $\Rightarrow$ (iii) of Theorem 3.2 is contained in a theorem of H. Lin, [L3, Theorem 5.4]. It also follows from [ELP, Theorem 3.1] after realizing that the conditions on $a$ in (i) and (ii) imply that the map $K_{1}\left(C^{*}(a, 1)\right) \rightarrow K_{1}(A)$, induced by the inclusion map, is zero. The implication (ii) $\Rightarrow$ (i) follows easily from the fact that $\mathbb{C} \backslash \Gamma_{\varepsilon}$ is dense in $\mathbb{C}$. The implication (iii) $\Rightarrow$ (i) is also easy — see also the proof of Proposition 3.1 above.

One could alternatively prove (ii) $\Rightarrow$ (iii) by mimicking the proof of Lemma 2.7. One would for this approach need Lin's result, $[\mathbf{L} 1]$, that if $A$ is a unital $\mathrm{C}^{*}$-algebra of real rank zero, then every unitary $u \in \mathcal{U}_{0}(A)$ can be approximated by unitaries with finite spectra. To follow the proof of Lemma 2.7 we would need actual spectral projections for $u$. They will in general not be available. Instead we can find projections, that approximately commute with $u$ and that approximately divide the spectrum of $u$ into two disjoint subsets. With some care, one can complete the proof of Lemma 2.7 in this fashion.

The proof of (i) $\Rightarrow$ (ii) is contained in the three lemmas below:

Lemma 3.3 (cf. [Rø1, Theorem 2.2]). Let $A$ be a unital $C^{*}$-algebra and let $a$ be an element in the closure of $\mathrm{GL}_{0}(A)$. Let $a=v|a|$ be the polar decomposition of $a$, with $v$ a partial isometry in $A^{* *}$. For each continuous function $f: \mathbb{R}^{+} \rightarrow \mathbb{R}^{+}$, such that $\left.f\right|_{[0, \varepsilon]} \equiv 0$ for some $\varepsilon>0$, there exists a unitary $u \in \mathcal{U}_{0}(A)$ such that $v f(|a|)=u f(|a|)$. 
Proof. This follows from [Rø1, Theorem 2.2] except for the part about the unitary $u$ lying in the connected component of the identity (and the assumption that $a$ lies in the closure of $\mathrm{GL}_{0}(A)$ rather than in the closure of $\mathrm{GL}(A))$. As we shall indicate below, the proof of [Rø1, Theorem 2.2] with obvious modifications - yields the claimed lemma.

We shift to the notation of $[\mathbf{R} \varnothing \mathbf{1}]$, and denote our $\mathrm{C}^{*}$-algebra by $\mathfrak{A}$, and the element $a \in A$ will be denoted by $T$ (so that $\alpha(T)=0$ - the distance from $T$ to the invertibles of $\mathfrak{A}$ is zero), and $T=V|T|$ is the polar decomposition of $T$.

In the proof of [Rø1, Theorem 2.1] choose the invertible element $A$ to lie in $\mathrm{GL}_{0}(\mathfrak{A})$ (which is possible by the assumption that $T$ lies in the closure of $\left.\mathrm{GL}_{0}(\mathfrak{A})\right)$. Then the element $S$ produced in that theorem will lie in $\mathrm{GL}_{0}(\mathfrak{A})$. The element $S_{0}$ constructed in [Rø1, Lemma 2.3] is homotopic to $S$, and will therefore also lie in $\mathrm{GL}_{0}(\mathfrak{A})$. To see this, go into the proof of $[\mathbf{R} \varnothing \mathbf{1}$, Lemma 2.3], put $D_{t}=S-t g\left(\left|T^{*}\right|\right) S+t S g(|T|)$ and put $E_{t}=S^{-1}-t g(|T|) S^{-1}+$ $t S^{-1} g\left(\left|T^{*}\right|\right)$. Then, following the proof, one finds that $D_{t} E_{t}=E_{t} D_{t}=1$, $D_{0}=S$, and $D_{1}=S_{0}$. Finally, the unitary $U$ found in $[\mathbf{R} \varnothing \mathbf{1}$, Theorem 2.2], which satisfies $U f(|T|)=V f(|T|)$, is equal to $S_{0}\left|S_{0}\right|^{-1}$, and so $U$ is homotopic to $S_{0}$ in $\mathrm{GL}_{0}(\mathfrak{A})$, and hence $U \in \mathcal{U}_{0}(\mathfrak{A})$.

The next lemma is an improvement of Lemma 2.2. Recall the definition of the functions $f_{\varepsilon}: \mathbb{R}^{+} \rightarrow \mathbb{R}^{+}$from above Lemma 2.2. If $f: \mathbb{R}^{+} \rightarrow \mathbb{R}^{+}$ is a continuous function with $f(0)=0$, then define a continuous function $\tilde{f}: \mathbb{C} \rightarrow \mathbb{C}$ by $\tilde{f}\left(r e^{i \theta}\right)=f(r) e^{i \theta}$.

Lemma 3.4. Let $A$ be a unital $C^{*}$-algebra, let a be a normal element in $A$, and let $a=v|a|$ be a polar decomposition of $a$, where $v \in A^{* *}$ is a partial isometry and $|a|=\left(a^{*} a\right)^{1 / 2}$.

(i) If $f: \mathbb{R}^{+} \rightarrow \mathbb{R}^{+}$is continuous with $f(0)=0$, then $v f(|a|)=\tilde{f}(a)$.

(ii) If $v f_{\varepsilon}(|a|)=u f_{\varepsilon}(|a|)$ for some unitary $u \in A$, and if $b=u\left(f_{\varepsilon}(|a|)+\right.$ $\varepsilon \cdot 1)$, then $b$ is normal and invertible, and $\|a-b\| \leq 2 \varepsilon$. Moreover, if $g: \mathbb{C} \rightarrow \mathbb{C}$ is a continuous function which is constant on $B(0, \varepsilon)$, then $g(a)=g(b)$, and

$$
\operatorname{sp}(b) \backslash \varepsilon \mathbb{T}=\operatorname{sp}(a) \backslash B(0, \varepsilon) .
$$

Proof. (i). This follows easily by approximating $f$ with functions $f_{0}$ of the form $f_{0}(r)=r h(r)$, where $h: \mathbb{R}^{+} \rightarrow \mathbb{R}^{+}$is continuous (not necessarily with $h(0)=0)$.

(ii). That $b$ is normal, and $\|a-b\| \leq 2 \varepsilon$ can be seen as in the proof of Lemma 2.2. Notice that $|b|=f_{\varepsilon}(|a|)+\varepsilon \cdot 1$. This shows that $|b|$ - and hence $b$ - are invertible, and that the spectrum of $b$ does not intersect the open ball with center 0 and radius $\varepsilon$. 
We shall apply the Borel function calculus inside the von Neumann algebra $A^{* *}$. Denoting the indicator function of the (Borel) set $E$ by $1_{E}$, set $e_{\varepsilon}=$ $1_{[0, \varepsilon]}(|a|)=1_{[0, \varepsilon]}(|b|)=1_{\{\varepsilon\}}(|b|)$. Put $\varphi(t)=t \cdot 1_{(\varepsilon, \infty)}(t)=\left(f_{\varepsilon}(t)+\varepsilon\right) \cdot 1_{(\varepsilon, \infty)}(t)$ for $t \in \mathbb{R}^{+}$.

$$
|a|\left(1-e_{\varepsilon}\right)=\varphi(|a|)=|b|\left(1-e_{\varepsilon}\right) .
$$

Since $e_{\varepsilon}$ commutes with $|b|$,

$$
a\left(1-e_{\varepsilon}\right)=v|a|\left(1-e_{\varepsilon}\right)=v\left(1-e_{\varepsilon}\right)|b|=u\left(1-e_{\varepsilon}\right)|b|=b\left(1-e_{\varepsilon}\right) .
$$

Now using that $e_{\varepsilon}$ commutes with $a$ and with $b$, we get

$$
g(a)\left(1-e_{\varepsilon}\right)=g\left(a\left(1-e_{\varepsilon}\right)\right)=g\left(b\left(1-e_{\varepsilon}\right)\right)=g(b)\left(1-e_{\varepsilon}\right)
$$

for every continuous function $g: \mathbb{C} \rightarrow \mathbb{C}$.

Assume that $g$ is constant on $B(0, \varepsilon)$. Put $\psi(z)=g(z) 1_{[0, \varepsilon]}(|z|)=$ $g(0) 1_{[0, \varepsilon]}(|z|)$. Then

$$
g(a) e_{\varepsilon}=\psi(a)=g(0) e_{\varepsilon}=\psi(b)=g(b) e_{\varepsilon} .
$$

In conclusion, we have shown that $g(a)=g(b)$. The claim about the spectra follows from the previous statement.

We now show an analogue of Lemma 2.4.

Lemma 3.5. Let $A$ be a unital $C^{*}$-algebra, let a be a normal element in $A$, and let $F$ be a finite subset of $\mathbb{C}$. If $a-\lambda \cdot 1$ lies in the closure of $\mathrm{GL}_{0}(A)$ for all $\lambda \in F$, then for every $\varepsilon>0$ there exists a normal element $b$ in $A$ with $\|a-b\| \leq \varepsilon$ and $b-\lambda \cdot 1 \in \mathrm{GL}_{0}(A)$ for every $\lambda \in F$.

Proof. Write $F=\left\{\lambda_{1}, \lambda_{2}, \ldots, \lambda_{n}\right\}$. We find successively normal elements $a_{0}=a, a_{1}, a_{2}, \ldots, a_{n}$ in $A$ satisfying

- $\left\|a_{j+1}-a_{j}\right\| \leq \varepsilon / n$,

- $a_{j}-\lambda_{i} \cdot 1 \in \mathrm{GL}_{0}(A)$ for $i=1,2, \ldots, j$,

- $a_{j}-\lambda_{i} \cdot 1$ lie in the closure of $\mathrm{GL}_{0}(A)$ for $i=j+1, j+2, \ldots, n$.

The element $b=a_{n}$ will then be as desired.

Assume $a_{j-1}$ has been found and that $1 \leq j \leq n$. Choose $\delta>0$ such that

- $\delta<\varepsilon / 2 n$,

- for every $c \in A$ with $\left\|c-a_{j-1}\right\| \leq \delta$, we have $c-\lambda_{i} \cdot 1 \in \mathrm{GL}_{0}(A)$ for $i=1,2, \ldots, j-1$.

- $\left|\lambda_{i}-\lambda_{j}\right|>\delta$ for $i=j+1, j+2, \ldots, n$,

Write $a_{j-1}-\lambda_{j} \cdot 1=v\left|a_{j-1}-\lambda_{j} \cdot 1\right|$, with $v$ a partial isometry in $A^{* *}$, and use Lemma 3.3 to find a unitary $u \in \mathcal{U}_{0}(A)$ so that $v f_{\delta}\left(\left|a_{j-1}-\lambda_{j} \cdot 1\right|\right)=$ $u f_{\delta}\left(\left|a_{j-1}-\lambda_{j} \cdot 1\right|\right)$. Put

$$
a_{j}=u\left(f_{\delta}\left(\left|a_{j-1}-\lambda_{j} \cdot 1\right|\right)+\delta \cdot 1\right)+\lambda_{j} \cdot 1 .
$$


It then follows from Lemma 3.4 (ii) that $a_{j}$ is normal, that $a_{j}-\lambda_{j} \cdot 1 \in \mathrm{GL}_{0}(A)$, and that $\left\|a_{j}-a_{j-1}\right\| \leq 2 \delta<\varepsilon / n$. By the choice of $\delta$ this implies that $a_{j}-\lambda_{i} \cdot 1 \in \mathrm{GL}_{0}(A)$ for $i=1,2, \ldots, j$.

Let $i \in\{j+1, j+2, \ldots, n\}$. We show that $a_{j}-\lambda_{i} \cdot 1$ is in the closure of $\mathrm{GL}_{0}(A)$. By the choice of $\delta$, we have that $\lambda_{i} \notin B\left(\lambda_{j}, \delta\right)$. We can therefore find continuous functions $f, g: \mathbb{C} \rightarrow \mathbb{C}$ such that

- $f(z) g(z)=z-\lambda_{i}$ for all $z \in \mathbb{C}$,

- $\left.f\right|_{B\left(\lambda_{j}, \delta\right)}$ is constant,

- $g(z)=\exp (h(z))$ for some continuous function $h: \mathbb{C} \rightarrow \mathbb{C}$.

The property of $g$ entails that $g(b) \in \mathrm{GL}_{0}(A)$ for every normal element $b \in A$. From Lemma 3.4 (ii) we conclude that $f\left(a_{j-1}\right)=f\left(a_{j}\right)$. Hence

$$
a_{j}-\lambda_{i} \cdot 1=f\left(a_{j}\right) g\left(a_{j}\right)=f\left(a_{j-1}\right) g\left(a_{j}\right)=\left(a_{j-1}-\lambda_{i} \cdot 1\right) g\left(a_{j-1}\right)^{-1} g\left(a_{j}\right) .
$$

By assumption, $a_{j-1}-\lambda_{i} \cdot 1$ lies in the closure of $\mathrm{GL}_{0}(A)$, and this shows that $a_{j}-\lambda_{i} \cdot 1$ lies in the closure of $\mathrm{GL}_{0}(A)$.

Proof of (i) $\Rightarrow$ (ii) in Theorem 3.2. Copy the proof of Lemma 2.5 using Lemma 3.5 instead of Lemma 2.4.

Recall from $[\mathbf{F R}]$ that a unital $\mathrm{C}^{*}$-algebra $A$ is said to have property (IN) if every normal element belongs to the closure of $\mathrm{GL}(A)$. A non-unital $\mathrm{C}^{*}$-algebra $A$ has property (IN) if its unitization $\tilde{A}$ has property (IN).

Definition 3.6. We say that a unital $\mathrm{C}^{*}$-algebra $A$ has property $\left(\mathrm{IN}_{0}\right)$ if every normal element that has 0 as an interior point of its spectrum lies in the closure of $\mathrm{GL}_{0}(A)$.

A non-unital $\mathrm{C}^{*}$-algebra $A$ is said to have property $\left(\mathrm{IN}_{0}\right)$ if $\tilde{A}$ has property $\left(\mathrm{IN}_{0}\right)$.

It is clear that property $\left(\mathrm{IN}_{0}\right)$ implies property (IN). Property (IN) does not imply $\left(\mathrm{IN}_{0}\right)$, not even for $\mathrm{C}^{*}$-algebras of real rank zero and stable rank one as Example 3.7 below shows.

Examples of $\mathrm{C}^{*}$-algebras satisfying $\left(\mathrm{IN}_{0}\right)$ are given in Proposition 3.8. The reader may prefer to consider the slightly more restrictive condition $\left(\mathrm{IN}_{00}\right)$ of a unital $\mathrm{C}^{*}$-algebra $A$, defined by requiring all normal non-invertible elements of $A$ to belong to the closure of $\mathrm{GL}_{0}(A)$. Trivially, $\left(\mathrm{IN}_{00}\right)$ implies $\left(\mathrm{IN}_{0}\right)$, but Example 3.9 gives a $\mathrm{C}^{*}$-algebra of real rank zero for which the reverse implication does not hold.

Example 3.7. Property (IN) does not imply property $\left(\mathrm{IN}_{0}\right)$.

Let $B_{1}, B_{2}$ be two unital $\mathrm{C}^{*}$-algebras of stable rank one, real rank zero, so that the unitary group of $B_{1}$ is disconnected, and ( $B_{1}$ and) $B_{2}$ are nonscattered. (One could for example take $B_{1}=B_{2}$ to be an irrational rotation $\mathrm{C}^{*}$-algebra.) Let $A$ be the $\mathrm{C}^{*}$-algebra $B_{1} \oplus B_{2}$. Then $A$ is unital, $\operatorname{sr}(A)=1$ and $\operatorname{RR}(A)=0$. 
Choose normal elements $b_{1} \in B_{1}$ and $b_{2} \in B_{2}$ with

$$
\operatorname{sp}\left(b_{1}\right)=\{z \in \mathbb{C}: 1 / 2 \leq|z| \leq 1\}, \quad \operatorname{sp}\left(b_{2}\right)=\{z \in \mathbb{C}:|z| \leq 1 / 2\},
$$

and with $b_{1} \notin \mathrm{GL}_{0}\left(B_{1}\right)$. Set $a=\left(b_{1}, b_{2}\right) \in A$. Then $\operatorname{sp}(a)=\mathbb{D}$, the closed unit disc in the complex plane. Hence $a-\lambda \cdot 1 \in \mathrm{GL}_{0}(A)$ for every $\lambda \in \mathbb{C} \backslash \operatorname{sp}(a)$ (because $\mathbb{C} \backslash \operatorname{sp}(a)$ is connected). But $a$ does not belong to the closure of $\mathrm{GL}_{0}(A)$, since $b_{1}$ does not belong to the closure of $\mathrm{GL}_{0}\left(B_{1}\right)$.

Hence $A$ does not have property ( $\left.\mathrm{IN}_{0}\right)$, but $\operatorname{sr}(A)=1$ (so $A$ has property $(\mathrm{IN})$ ), and $\operatorname{RR}(A)=0$.

Proposition 3.8. Every simple unital $C^{*}$-algebra, which has stable rank one or is purely infinite, has property $\left(\mathrm{IN}_{0}\right)$.

Proof. The two classes of $\mathrm{C}^{*}$-algebras in the proposition have in common that they have property (IN) (see [Rø2, Theorem 4.4]), and if $B$ is a non-zero hereditary sub-C*-algebra of $A$, then the natural map $\mathcal{U}(\tilde{B}) \rightarrow \mathcal{U}(A) / \mathcal{U}_{0}(A)$ is surjective (see $[\mathbf{R i}$, Theorem 10.10] and [C, Theorem 1.9]).

Let $a$ be a normal non-invertible element of $A$, and let $\varepsilon>0$. Let $a=v|a|$ be the polar decomposition for $a$, with $v \in A^{* *}$, and recall from $[\mathbf{R} \varnothing 1$, Theorem 2.1] that there exists a unitary $u \in A$ so that $v f_{\varepsilon}(|a|)=u f_{\varepsilon}(|a|)$. Let $g: \mathbb{R}^{+} \rightarrow \mathbb{R}^{+}$be a continuous function that vanishes on $[\varepsilon, \infty)$, and with $g(0)=1$. Then $g(|a|) \neq 0$ because $a$ is non-invertible, and we can therefore find a unitary $w \in \overline{g(|a|) A g(|a|)}+1$ such that $u w \sim_{h} 1$. Set $b=u w\left(f_{\varepsilon}(|a|)+\varepsilon \cdot 1\right.$ ). Then $b \in \mathrm{GL}_{0}(A)$ (we are not claiming here that $b$ is normal), and

$\|a-b\| \leq\left\|a-u\left(f_{\varepsilon}(|a|)+\varepsilon \cdot 1\right)\right\|+\left\|w\left(f_{\varepsilon}(|a|)+\varepsilon \cdot 1\right)-\left(f_{\varepsilon}(|a|)+\varepsilon \cdot 1\right)\right\| \leq 4 \varepsilon$, where we have used that $w f_{\varepsilon}(|a|)=f_{\varepsilon}(|a|)$.

This argument shows that $A$ actually has property $\left(\mathrm{IN}_{00}\right)$.

Villadsen has found an example of a simple $\mathrm{C}^{*}$-algebra with stable rank 2 , showing that a specific normal element of the constructed $\mathrm{C}^{*}$-algebra cannot be approximated by invertible elements (see $[\mathbf{V}]$ ). This example is therefore a simple, stably finite $\mathrm{C}^{*}$-algebra that does not have property (IN), and hence neither $\left(\mathrm{IN}_{0}\right)$ nor $\left(\mathrm{IN}_{00}\right)$.

Example 3.9. Property $\left(\mathrm{IN}_{0}\right)$ does not imply property $\left(\mathrm{IN}_{00}\right)$.

Let $B$ be any real rank zero, unital $\mathrm{C}^{*}$-algebra that has property $\left(\mathrm{IN}_{0}\right)$ and non-connected group of unitary elements, and set $A=B \oplus \mathbb{C}$. (Here $B$ could be an irrational rotation $\mathrm{C}^{*}$-algebra, cf. Proposition 3.8.) If zero is an interior point of the spectrum of $a=(b, \lambda) \in A$, then zero is an interior point of the spectrum of $b$. Hence $b$ lies in the closure of $\mathrm{GL}_{0}(B)$, and from this we get that $a$ lies in the closure of $\mathrm{GL}_{0}(A)$.

The $\mathrm{C}^{*}$-algebra $A$ does not have property $\left(\mathrm{IN}_{00}\right)$. Indeed, if $u \in \mathcal{U}(B) \backslash$ $\mathcal{U}_{0}(B)$, and if $a=(u, 0) \in A$, then $a$ is normal and non-invertible, but $a$ is not in the closure of $\mathrm{GL}_{0}(A)$. 
Corollary 3.10. Let $A$ be a unital $C^{*}$-algebra. The following two conditions are equivalent:

(i) Every normal element $a \in A$, satisfying

$$
a-\lambda \cdot 1 \in \mathrm{GL}_{0}(A)
$$

for all $\lambda \in \mathbb{C} \backslash \operatorname{sp}(a)$, is the norm-limit of normal elements in $A$ with finite spectrum.

(ii) A has real rank zero and property $\left(I N_{0}\right)$.

Proof. (i) $\Rightarrow$ (ii). Assume that (i) holds. Every self-adjoint element $a$ in $A$ is normal and satisfies $a-\lambda \cdot 1 \in \mathrm{GL}_{0}(A)$ for all $\lambda \in \mathbb{C} \backslash \operatorname{sp}(a)$ (because $\mathbb{C} \backslash \operatorname{sp}(a)$ is connected). Hence $a$ can be approximated within any given tolerance by a normal element $b$ with finite spectrum. It is easily seen, that $\left(b+b^{*}\right) / 2$ is a self-adjoint element with finite spectrum whose distance to $a$ is at most $\|a-b\|$. This shows that $A$ has real rank zero.

We proceed to show that $A$ has property $\left(\mathrm{IN}_{0}\right)$. Let $a$ be a normal element in $A$, and assume that there exists an $r>0$ such that $B(0, r) \subseteq \operatorname{sp}(a)$. Let $a=v|a|$ be the polar decomposition of $a$, with $v$ a partial isometry in $A^{* *}$.

Consider the continuous functions $f: \mathbb{R}^{+} \rightarrow \mathbb{R}^{+}, g: \mathbb{R}^{+} \rightarrow \mathbb{R}^{+}$, given by $f(t)=\min \left\{r^{-1} t, 1\right\}$ and $g(t)=\max \{r, t\}$, and $h=\tilde{f}: \mathbb{C} \rightarrow \mathbb{C}$ (see above Lemma 3.4). Notice that $f(t) g(t)=t$, and that $v f(|a|)=h(a)$ (by Lemma 3.4 (i)).

The element $h(a)$ is therefore normal and $\operatorname{sp}(h(a))=h(\operatorname{sp}(a))=\mathbb{D}$ (the closed unit disc in the complex plane). Consequently, $h(a)-\lambda \cdot 1 \in \mathrm{GL}_{0}(A)$ for all $\lambda \in \mathbb{C} \backslash \operatorname{sp}(h(a))$ (because $\mathbb{C} \backslash \operatorname{sp}(h(a))$ is connected). Assuming (i), we conclude that $h(a)$ can be approximated by normal elements with finite spectra, and therefore $h(a)$ lies in the closure of $\mathrm{GL}_{0}(A)$ (cf. the proof of Proposition 3.1). Since $a=v f(|a|) g(|a|)=h(a) g(|a|)$, and since $g(|a|) \in$ $\mathrm{GL}_{0}(A)$, we conclude that $a$ lies in the closure of $\mathrm{GL}_{0}(A)$. It has now been proved that $A$ has property $\left(\mathrm{IN}_{0}\right)$.

(ii) $\Rightarrow$ (i). By Theorem 3.2 it suffices to show that $a-\lambda \cdot 1$ lies in the closure of $\operatorname{GL}_{0}(A)$ for all $\lambda \in \mathbb{C}$. This is the case by assumption on $a$ if $\lambda \notin \operatorname{sp}(a)$. By continuity, $a-\lambda \cdot 1$ lies in the closure of $\mathrm{GL}_{0}(A)$ for all $\lambda$ in the closure of $\mathbb{C} \backslash \operatorname{sp}(a)$. The remaining points, $\lambda$, are the interior points of the spectrum of $a$, and there $a-\lambda \cdot 1$ is in the closure of $\mathrm{GL}_{0}(A)$ by the assumption that $A$ has property $\left(\mathrm{IN}_{0}\right)$.

Corollary 3.11. Let $A$ be a unital $C^{*}$-algebra. Assume that $\operatorname{RR}(A)=0$, that $A$ has property (IN), and that

(NT) for every hereditary sub-C ${ }^{*}$-algebra $B$ of $A$, the map

$$
\mathcal{U}(\tilde{B}) \rightarrow \mathcal{U}(\tilde{I}) / \mathcal{U}_{0}(\tilde{I}),
$$

where $I$ is the ideal of $A$ generated by $B$, is surjective. 
Then every normal element $a \in A$, satisfying

$$
\pi_{I}(a)-\lambda \cdot 1 \in \mathrm{GL}_{0}(A / I)
$$

for every proper ideal $I$ of $A$, and for every $\lambda \in \mathbb{C} \backslash \operatorname{sp}\left(\pi_{I}(a)\right)$, is a norm limit of normal elements in A with finite spectra.

Proof. By Theorem 3.2 it suffices to show that every normal element $a \in A$, satisfying

$$
\pi_{I}(a) \in \mathrm{GL}(A / I) \Rightarrow \pi_{I}(a) \in \mathrm{GL}_{0}(A / I),
$$

lies in the closure of $\mathrm{GL}_{0}(A)$. Let $\varepsilon>0$. Write $a=v|a|$ with $v$ a partial isometry in $A^{* *}$. By the assumption that $A$ has property (IN), and by Lemma 3.3, there is a unitary $u \in A$ so that $v f_{\varepsilon}(|a|)=u f_{\varepsilon}(|a|)$. We wish to replace $u$ by another unitary that belongs to $\mathcal{U}_{0}(A)$. Since $A$ is of real rank zero, we can find a projection $p$ in the hereditary subalgebra of $A$ generated by $f_{\varepsilon}(|a|)$ so that $\left\|(1-p) f_{\varepsilon}(|a|)\right\| \leq \varepsilon$. We show that there is a unitary $v \in(1-p) A(1-p)$ such that $u^{*} \sim_{h} p+v$ in $\mathcal{U}(A)$. This will imply that $b=u\left(p+v^{*}\right)\left(f_{\varepsilon}(|a|)+\varepsilon \cdot 1\right) \in \mathrm{GL}_{0}(A)$, and

$$
\begin{aligned}
&\|a-b\| \leq\left\|a-u\left(f_{\varepsilon}(|a|)+\varepsilon \cdot 1\right)\right\| \\
&+\left\|\left(p+v^{*}\right)\left(f_{\varepsilon}(|a|)+\varepsilon \cdot 1\right)-\left(f_{\varepsilon}(|a|)+\varepsilon \cdot 1\right)\right\| \\
& \leq 2 \varepsilon+\left\|\left(v^{*}-(1-p)\right)\left(f_{\varepsilon}(|a|)+\varepsilon \cdot 1\right)\right\| \leq 6 \varepsilon .
\end{aligned}
$$

Let $I$ be the closed two-sided ideal in $A$ generated by $1-p$. If $I=A$, then $(1-p) A(1-p)$ contains a unitary $v$ with $u^{*} \sim_{h} p+v$ in $\mathcal{U}(A)$ by the assumption (NT). Assume that $I \neq A$. Because $p$ lies in the hereditary subalgebra generated by $f_{\varepsilon}(|a|)$, we have $p|a| p \geq \varepsilon p$; this entails $\pi_{I}(|a|) \geq \varepsilon \cdot 1$, and so $\pi_{I}(a) \in \operatorname{GL}(A / I)$, which by the assumption on $a$ implies $\pi_{I}(a) \in$ $\mathrm{GL}_{0}(A / I)$. It follows that

$$
\pi_{I}(u) \sim_{h} \pi_{I}\left(u f_{\varepsilon}(|a|)\right) \sim_{h} \pi_{I}(a) \sim_{h} 1
$$

in $\operatorname{GL}(A / I)$. We can therefore find $w \in \mathcal{U}_{0}(A)$ with $\pi_{I}(w)=\pi_{I}(u)$. Since $w^{*} u \in \mathcal{U}(\tilde{I})$ it follows from the assumption (NT) that there exists a unitary $v$ in $(1-p) A(1-p)$ with $\left(w^{*} u\right)^{*} \sim_{h} p+v$. This completes the proof, because $w^{*} u \sim_{h} u$.

Every $\mathrm{C}^{*}$-algebra of stable rank one has property (IN) (for trivial reasons), and also property (NT) (for less trivial reasons). For the latter one can use $[\mathbf{R i}$, Theorem 10.10]. With these observations we get the following corollary to Corollary 3.11:

Corollary 3.12. Let $A$ be a unital $C^{*}$-algebra of real rank zero and stable rank one. Then every normal element $a \in A$, satisfying

$$
\pi_{I}(a)-\lambda \cdot 1 \in \mathrm{GL}_{0}(A / I)
$$

for every proper ideal $I$ of $A$ and every $\lambda \in \mathbb{C} \backslash \operatorname{sp}\left(\pi_{I}(a)\right)$, is a norm limit of normal elements in $A$ with finite spectra. 
Corollary 3.12 can for example be applied to the real rank zero ATalgebras classified by George Elliott in $[\mathbf{E}]$. (AT-algebras is the class of $\mathrm{C}^{*}$-algebras obtained from the $\mathrm{C}^{*}$-algebra $C(\mathbb{T})$ with the operations of tensoring by $M_{n}(\mathbb{C})$, taking direct sums, and taking inductive limits.)

It would be interesting to know if one can replace the assumption in Corollary 3.12 that $\operatorname{sr}(A)=1$ with the weaker assumption that $A$ has property (IR) (cf. [FR, 3.1] and the main theorem of [L4]). By Corollary 3.11 that would be the case if property (IR) (together with $\mathrm{RR}(A)=0$ ) implies property (NT). This is known to be true for simple $\mathrm{C}^{*}$-algebra, because a simple unital $\mathrm{C}^{*}$-algebra $A$ has property (IR) if and only if either $\operatorname{sr}(A)=1$ or $A$ is purely infinite.

Example 3.13. Of the three sufficient conditions in Corollary 3.11, the real rank zero condition is clearly also necessary (cf. the proof of Corollary 3.10). It is possible that the condition (NT) always holds for real rank zero $\mathrm{C}^{*}$ algebras. Larry Brown has informed us that examples of $\mathrm{C}^{*}$-algebras of real rank zero, which do not have property (IN), exist. Condition (NT) is necessary in Corollary 3.11, at least when $A$ is simple, as the following example shows:

Assume that $A$ is a simple unital $\mathrm{C}^{*}$-algebra of real rank zero where property (NT) of Corollary 3.11 does not hold - if such an example exists. Since every hereditary sub-C*-algebra of $A$ is the inductive limit of corner algebras $p A p$, where $p$ is a projection in $A$, there is a unitary $u \in A$ and a projection $p \in A$ with the property that there is no unitary $v \in p A p$ satisfying $u \sim_{h} v+(1-p)$. Since $A$ is of real rank zero, and $\operatorname{since} \operatorname{sp}(u)=\mathbb{T}$, there is a non-zero projection $q \in A$ such that $\|q u q-q\| \leq 1 / 2$. Then $(1-q) u(1-q)$ is invertible in $(1-q) A(1-q)$, and $z=q+(1-q) u(1-q)$ is homotopic to $u$ in $\mathrm{GL}_{0}(A)$. Put $u_{0}=z|z|^{-1}$. Then $u_{0} \in \mathcal{U}(A), u \sim_{h} u_{0}$ and $q u_{0}=u_{0} q=q$.

Let $x$ be a non-zero element in $q A p$, and let $e$ be a non-zero projection in $\overline{x A x^{*}}$. Then $e \leq q$ and $e \precsim p$. It follows that $e u_{0} e=e$ and that there is no unitary $v \in e A e$ such that $u_{0} \sim_{h} v+(1-e)$. The corner algebra $e A e$ is non-scattered (because $A$ must be infinite-dimensional), and we can therefore find a normal element $c \in e A e$ with $\operatorname{sp}(c)=\mathbb{D}$.

Put $a=c+(1-e) u_{0}(1-e)$. Then $a$ is normal and $\operatorname{sp}(a)=\mathbb{D}$, and so $a-\lambda \cdot 1 \in \mathrm{GL}_{0}(A)$ for every $\lambda \in \mathbb{C} \backslash \operatorname{sp}(a)$. We claim that $a$ is not in the closure of $\mathrm{GL}_{0}(A)$, and this will show that $a$ cannot be approximated by normal elements with finite spectra, cf. Theorem 3.2. Indeed, assume that $b \in \mathrm{GL}_{0}(A)$ and that $\|a-b\|<1$. Then

$$
\left\|(1-e) u_{0}(1-e)-(1-e) b(1-e)\right\|<1,
$$

and $(1-e) u_{0}(1-e)$ is unitary in $(1-e) A(1-e)$. Hence $(1-e) b(1-e)$ is invertible in $(1-e) A(1-e)$ with an inverse we denote by $r$. As in a standard 
$2 \times 2$ matrix trick,

$(\mathbf{9})$

$$
\begin{aligned}
(1-e) b(1-e)+(e b e-e b r b e) & =(1-e b r(1-e)) b(1-(1-e) r b e) \\
& \sim_{h} b
\end{aligned}
$$

in $\operatorname{GL}(A)$. Hence $d=e b e-e b r b e \in \operatorname{GL}(e A e)$ and so $d=v^{*}|d|$ for some unitary $v \in e A e$. By (\$) we also have

$$
\begin{aligned}
(1-e)+v \sim_{h}(1-e)+d^{-1} & \sim_{h}(1-e) b(1-e)+e \\
& \sim_{h}(1-e) u_{0}(1-e)+e=u_{0}
\end{aligned}
$$

in contradiction with the stipulated properties of $u_{0}$ and $e$.

\section{References}

[BD] I.D. Berg and K.R. Davidson, Almost commuting matrices and a quantative version of the Brown-Douglas-Fillmore theorem, Acta Math., 166 (1991), 121-161, MR 92f:47015, Zbl 731.47009.

[BDF1] L.G. Brown, R. Douglas and P. Fillmore, Unitary equivalence modulo the compact operators and extensions of $C^{*}$-algebras, in 'Proc. Conf. on Operator Theory', Lecture notes in Math., 345, Springer-Verlag, Heidelberg, (1973), 58-128, MR 52 \#1378, Zbl 277.46053.

[BDF2] , Extensions of $C^{*}$-algebras and K-homology, Ann. of Math., 105 (1977), 265-324, MR 56 \#16399, Zbl 376.46036.

[C] J. Cuntz, K-theory for certain $C^{*}$-algebras, Ann. of Math., 113 (1981), 181-197, MR 84c:46058, Zbl 446.46052.

[D] A.M. Davie, Classification of essentially normal operators, in 'Spaces of Analytic Functions', Lecture notes in Math., 512, Springer-Verlag, Heidelberg, (1976), 31-55, MR 58 \#12478, Zbl 337.47010.

[ELP] S. Eilers, T. Loring and G.K. Pedersen, Fragility for subhomogeneous $C^{*}$-algebras with one-dimensional spectrum, Bull. London. Math. Soc., 31(3) (1999), 337-344, MR 2000a:46092.

[E] G.A. Elliott, On the classification of $C^{*}$-algebras of real rank zero, J. Reine Angew. Math., 443 (1993), 179-219, MR 94i:46074, Zbl 809.46067.

[FR] P. Friis and M. Rørdam, Almost commuting self-adjoint matrices - A short proof of Huaxin Lin's theorem, J. Reine Angew. Math., 479 (1996), 121-131, MR 97i:46097, Zbl 859.47018.

[L1] H. Lin, Exponential rank of $C^{*}$-algebras with real rank zero and Brown-Pedersen's conjecture, J. Funct. Anal., 114 (1993), 1-11, MR 95a:46079, Zbl 812.46054.

[L2] _ Almost commuting self-adjoint matrices and applications, in Operator Algebras and their Applications, Fields Institute Communications, 13, (1995), 193-233, MR 98c:46121, Zbl 881.46042.

[L3] - Approximation by normal elements of finite spectra in $C^{*}$-algebras of real rank zero, Pacific J. Math., 173(2) (1996), 443-489, MR 98h:46059, Zbl 860.46039.

[L4] $\quad C^{*}$-algebras with weak $(F N)$, J. Funct. Anal., 150 (1997), 65-74, MR 98g:46084. 
[L5] The generalized Berg theorem and BDF-theorem, Trans. Amer. Math. Soc., 349 (1997), 529-545, MR 98g:46085, Zbl 870.46034.

[Ri] M.A. Rieffel, Dimension and stable rank in the $K$-theory of $C^{*}$-algebras, Proc. London Math. Soc., 46(3) (1983), 301-333, MR 84g:46085, Zbl 533.46046.

[Rø1] M. Rørdam, Advances in the theory of unitary rank and regular approximation, Ann. of Math., 128 (1988), 153-172, MR 90c:46072, Zbl 659.46052.

[Rø2] On the structure of simple $C^{*}$-algebras tensored with a UHF-algebra, J. Funct. Anal., 100 (1991), 1-17, MR 92m:46091, Zbl 773.46028.

[V] J. Villadsen, On the stable rank of simple $C^{*}$-algebras, J. Amer. Math. Soc., 12(4) (1999), 1091-1102, MR 2000f:46075, Zbl 937.46052.

Received November 1, 1998. The first author was supported by The Danish Research Academy.

Department of Mathematics

UNIVERSITY OF TORONTO

100 St. George Street

Toronto, Ontario M5S 3G1

CANADA

E-mail address: friis@math.toronto.edu

Department of Mathematics

University of COPENHAGEN

UNIVERSITETSPARKEN 5

2100 Copenhagen $\varnothing$

DENMARK

E-mail address: rordam@math.ku.dk 